\title{
Time-Accurate Computations of Isolated Circular Synthetic Jets in Crossflow
}

\author{
C. L. Rumsey, N. W. Schaeffler ${ }^{\dagger}$ \\ NASA Langley Research Center, Hampton, VA 23681-2199, USA \\ I. M. Milanovic ${ }^{\ddagger}$ \\ University of Hartford, West Hartford, CT 06117, USA \\ K. B. M. Q. Zaman ${ }^{\S}$ \\ NASA Glenn Research Center, Cleveland, $\mathrm{OH} 44135$, USA
}

\begin{abstract}
Results from unsteady Reynolds-averaged Navier-Stokes computations are described for two different synthetic jet flows issuing into a turbulent boundary layer crossflow through a circular orifice. In one case the jet effect is mostly contained within the boundary layer, while in the other case the jet effect extends beyond the boundary layer edge. Both cases have momentum flux ratios less than 2. Several numerical parameters are investigated, and some lessons learned regarding the CFD methods for computing these types of flow fields are outlined. Results in both cases are compared to experiment.
\end{abstract}

\section{Introduction}

Synthetic jets are attracting increasing interest in the aerospace community because they have many practical applications, including jet vectoring, control of separation, enhanced mixing, reduction of wall skin friction, and virtual aeroshaping. As a practical tool, they are generally more attractive than steady blowing or suction because they do not require any complex fluid ducting or plumbing systems. Furthermore, synthetic jets have been found to achieve similar effectiveness to steady blowing or suction with considerably smaller momentum (and hence lower energy inputs). ${ }^{1}$

The problem of synthetic jet flow in a turbulent boundary layer has been studied both experimentally (see, for example ${ }^{2-5}$ ) and computationally (see, for example ${ }^{6-11}$ ). Earlier numerical work by Rizzetta et al. ${ }^{12}$ for synthetic jets into quiescent flow demonstrated that accounting for the internal actuator geometry was important because it affected the jet profiles at the exit. Lee and Goldstein ${ }^{6}$ used direct numerical simulation (DNS) to study an array of synthetic microjets in a crossflow. A 4-slot array was modeled, and adjacent jets were coupled $180^{\circ}$ out of phase. As microjets, the Reynolds number of the jets was very small, on the order of 10 . Weak jets were confined to the linear sublayer, but when the jets penetrated up to the buffer region, they affected the events of the boundary layer significantly.

Mittal et al. ${ }^{7}$ and Mittal and Rampunggoon ${ }^{8}$ used 2-D Navier-Stokes to investigate the use of stronger synthetic jets immersed in a boundary layer to form large mean recirculation bubbles (for aeroshaping). They modeled the cavity, but their studies were purely numerical (there was no associated experiment). Similarly, Ravi et al. ${ }^{9}$ performed a purely numerical study of synthetic jet in crossflow. They also modeled the cavity, and used 3-D Navier-Stokes to investigate the effects of a square and two rectangular shaped slots.

Cui et al. ${ }^{10}$ performed 2-D computations on a synthetic jet in a turbulent crossflow boundary layer using unsteady Reynolds-averaged Navier-Stokes (URANS) with the Spalart-Allmaras turbulence model. ${ }^{13}$ They compared their computational fluid dynamics (CFD) results with two experiments: one in which the discrete vortices scaled with the boundary layer thickness, and one in which they scaled with the inner viscous sublayer. Overall, they found qualitatively good agreement with experiment, although in the latter case the computed vortices moved too slowly

\footnotetext{
* Senior Research Scientist, Computational Aerosciences Branch, Mail Stop 128, Associate Fellow AIAA.

${ }^{\dagger}$ Research Scientist, Flow Physics and Control Branch, Mail Stop 170, Member AIAA.

$¥$ Assistant Professor, Mechanical Engineering, 200 Bloomfield Ave., Member AIAA.

§Aerospace Engineer, Turbomachinery \& Propulsion Systems Division, Nozzle Branch, Mail Stop 86-7, Associate Fellow AIAA.

Copyright (c) 2005 by the American Institute of Aeronautics and Astronautics, Inc. The U.S. Government has a royalty-free license to exercise all rights under the copyright claimed herein for Governmental purposes. All other rights are reserved by the copyright owner.
} 
downstream. Also, in both cases there were significant quantitative differences between the CFD results and experiment.

A CFD validation workshop on synthetic jets and turbulent separation control (CFDVAL2004) ${ }^{14,15}$ was held in March 2004 in Williamsburg, VA. The purpose of the workshop was to assess the current capabilities of different classes of turbulent flow solution methodologies to predict flow fields induced by synthetic jets and separation control. Case 2 of the workshop was a synthetic jet issuing into a turbulent boundary layer through a circular orifice in the floor. This experiment was conducted at NASA Langley Research Center specifically for use as a CFD validation case. ${ }^{16} \mathrm{~A}$ similar experiment was also recently conducted at NASA Glenn Research Center. ${ }^{17}$

A summary of the CFD results for case 2 of the CFDVAL2004 workshop is given in Rumsey et al. ${ }^{15}$ There were five contributers who ran ten separate cases. One contributor used large eddy simulation (LES) and the others all used URANS. On the whole, reasonably good qualitative results were obtained compared with experiment both for longtime averaged and phase averaged quantities, but there were significant variations among the CFD results. Different turbulence models were found to have a much smaller effect than different grids, codes, and boundary conditions. Cui and Agarwal ${ }^{11}$ also computed case 2 from CFDVAL2004, using both URANS with Menter's SST turbulence model ${ }^{18}$ and detached eddy simulation (DES). ${ }^{19}$ The two methods produced overall similar results in qualitative agreement with experiment, and DES was able to simulate some of the larger cross-stream velocity components downstream.

The intent of the current paper is to summarize, compare, and contrast the CFD results obtained using the URANS code CFL3D ${ }^{20}$ for the two cases hereafter described as the "CFDVAL case" (Schaeffler and Jenkins ${ }^{16}$ ) and the "NASA Glenn case" (Milanovic et al. ${ }^{17}$ ). Previous CFD results using CFL3D were reported for the CFDVAL workshop case, ${ }^{21,22}$ but only limited results were reported for the NASA Glenn case in a paper devoted primarily to describing the experiment. ${ }^{17}$

\section{Numerical Method}

The computer code CFL3D ${ }^{20}$ solves the three-dimensional, time-dependent, Reynolds-averaged compressible Navier-Stokes equations with an upwind finite-volume formulation (it can also be exercised in two-dimensional mode of operation for 2-D cases). Because the code is compressible, the Navier-Stokes equations are averaged using Favre averaged variables; e.g., $\overline{\rho u_{i}} / \bar{\rho}$. CFL3D can solve flows over multiple-zone grids that are connected in a one-to-one, patched, or overset manner, and can employ grid sequencing, multigrid, and local time stepping when accelerating convergence to steady state. Upwind-biased spatial differencing is used for the inviscid terms, and flux limiting is used to obtain smooth solutions in the vicinity of shock waves, when present. Viscous terms are centrally differenced, and cross-diffusion terms are neglected. For very low Mach number flows, preconditioning ${ }^{23}$ is used to insure convergence and accuracy of the solutions.

The CFL3D code is advanced in time with an implicit approximate factorization method. The implicit derivatives are written as spatially first-order accurate, which results in block tridiagonal inversions for each sweep. However, for solutions that utilize Roe flux-difference splitting, ${ }^{24}$ the block tridiagonal inversions are further simplified using a diagonal algorithm with a spectral radius scaling of the viscous terms.

In time-accurate mode, CFL3D uses pseudo-time stepping with multigrid and achieves second order temporal accuracy. With pseudo-time stepping, subiterations are used to reduce the linearization and factorization errors, and advance the solution in pseudo-time to the next physical time. For a non-deforming mesh, the time dependent compressible Navier-Stokes equations can be written as

$$
\frac{1}{J} \frac{\partial \mathbf{Q}}{\partial t}=R(\mathbf{Q})
$$

where $\mathbf{Q}$ is the vector of conserved variables $[\rho, \rho u, \rho v, \rho w, e]^{T}, J$ is the Jacobian of the generalized coordinate transformation, and $R$ is the right-hand-side:

$$
R(\mathbf{Q})=-\left[\frac{\partial\left(\hat{\mathbf{F}}-\hat{\mathbf{F}}_{v}\right)}{\partial \xi}+\frac{\partial\left(\hat{\mathbf{G}}-\hat{\mathbf{G}}_{v}\right)}{\partial \eta}+\frac{\partial\left(\hat{\mathbf{H}}-\hat{\mathbf{H}}_{v}\right)}{\partial \zeta}\right]
$$

made up of gradients of the inviscid and viscous flux terms. Eq. (1) can be discretized with backward differencing:

$$
\frac{(1+\phi)\left(\mathbf{Q}^{n+1}-\mathbf{Q}^{n}\right)-\phi\left(\mathbf{Q}^{n}-\mathbf{Q}^{n-1}\right)}{J \Delta t}=R\left(\mathbf{Q}^{n+1}\right) .
$$

The superscripts $n, n+1$, and $n-1$ indicate the time level. When $\phi=0$ the method is first-order temporally accurate, and when $\phi=1 / 2$ the method is second-order temporally accurate. To employ pseudo-time stepping, a pseudo time 
term is added to Eq. (3), and the equation is iterated in $m$, where $m$ is the subiteration counter. After linearizing $R$ and performing some additional manipulation, one obtains:

$$
\begin{aligned}
& {\left[\left(\frac{1+\phi^{\prime}}{J \Delta \tau}+\frac{1+\phi}{J \Delta t}\right) \mathbf{I}+\partial_{\xi} \mathbf{A}+\partial_{\eta} \mathbf{B}+\partial_{\zeta} \mathbf{C}\right] \Delta \mathbf{Q}^{m}=} \\
& \frac{\phi^{\prime} \Delta \mathbf{Q}^{m-1}}{J \Delta \tau}+\frac{\phi \Delta \mathbf{Q}^{n-1}}{J \Delta t}-\frac{(1+\phi)\left(\mathbf{Q}^{m}-\mathbf{Q}^{n}\right)}{J \Delta t}+R\left(\mathbf{Q}^{m}\right)
\end{aligned}
$$

where $\tau$ is the pseudo time parameter, $\Delta \mathbf{Q}^{m}=\mathbf{Q}^{m+1}-\mathbf{Q}^{m}$, and

$$
\mathbf{A}=\frac{\partial\left(\hat{\mathbf{F}}-\hat{\mathbf{F}}_{v}\right)}{\partial \mathbf{Q}} \quad \mathbf{B}=\frac{\partial\left(\hat{\mathbf{G}}-\hat{\mathbf{G}}_{v}\right)}{\partial \mathbf{Q}} \quad \mathbf{C}=\frac{\partial\left(\hat{\mathbf{H}}-\hat{\mathbf{H}}_{v}\right)}{\partial \mathbf{Q}}
$$

Eq. (4) is approximately factored and written in primitive variable form; it is solved as a series of sweeps in each coordinate direction. Additional details are given in Krist et al. ${ }^{20}$ In the current study, the effects of different physical time steps and number of subiterations were investigated.

The turbulence models are solved uncoupled from the mean flow equations using implicit approximate factorization. Their advective terms are solved using first-order upwind differencing. Many turbulence models are available in CFL3D, but only those used in the current study are mentioned here. Descriptions of the one-equation SpalartAllmaras (SA) and the two-equation Menter $k-\omega$ shear-stress transport (SST) turbulence models can be found in their respective references. ${ }^{13,18}$ Both of these models are linear eddy-viscosity models that make use of the Boussinesq eddy-viscosity hypothesis. The EASM-ko model is a nonlinear eddy-viscosity model: it stands for explicit algebraic stress model in $k-\omega$ form. A detailed description of this model can be found in Rumsey and Gatski. ${ }^{25}$

For time-accurate URANS computations, it can be shown that the dependent variables calculated for forced periodic cases correspond to phase-averaged variables. Following Reynolds and Hussain, ${ }^{26}$ triple decomposition can be used to represent a fluctuating signal:

$$
f(\mathbf{x}, t)=\bar{f}(\mathbf{x})+\tilde{f}(\mathbf{x}, t)+f^{\prime}(\mathbf{x}, t)
$$

where $\bar{f}(\mathbf{x})$ is the (time) mean value, $\tilde{f}(\mathbf{x}, t)$ is the statistical contribution of the organized motion, and $f^{\prime}(\mathbf{x}, t)$ is the turbulence. The long-time average is defined as

$$
\bar{f}(\mathbf{x})=\lim _{T \rightarrow \infty} \frac{1}{T} \int_{0}^{T} f(\mathbf{x}, t) d t
$$

and the phase average is defined as

$$
\langle f(\mathbf{x}, t)\rangle=\lim _{N \rightarrow \infty} \frac{1}{N} \sum_{n=0}^{N} f(\mathbf{x}, t+n \tau)
$$

where $\tau$ in the case of a synthetic jet flow field is the period of the imposed oscillatory motion. Thus, the wave component is

$$
\tilde{f}(\mathbf{x}, t)=\langle f(\mathbf{x}, t)\rangle-\bar{f}(\mathbf{x})
$$

and the decomposition of Eq. 6 can be written as

$$
f(\mathbf{x}, \mathbf{t})=\langle f(\mathbf{x}, \mathbf{t})\rangle+f^{\prime}(\mathbf{x}, \mathbf{t}) .
$$

This form now resembles standard Reynolds splitting, except that the flow is split into a slowly varying mean (phase average) and a random fluctuating part. See also Gatski and Liu. ${ }^{27}$ As a result, the final conservation equations in terms of phase-averaged variables \langle\rangle are identical in form to the standard Reynolds-averaged equations. In other words, when URANS is used for a forced periodic flow field such as that produced by a synthetic jet, the computation typically eventually settles down to a nearly exactly repeatable periodic variation. If and when this repeatability is attained, any single point during the cycle corresponds to a phase-averaged result from the experiment. An example showing the cycle-to-cycle repeatability for a global integrated quantity is shown in Fig. 1 for the CFDVAL case. Here, coefficient of drag nondimensionalized by a unit reference length (the absolute level of which is not particularly meaningful in this case) is plotted as a function of iteration. An FFT analysis (not shown) over the last three periods 
confirms that repeatability has been obtained, with the coefficients of lower frequency modes more than 2 orders of magnitude lower than that of the driving frequency.

All of the results for the current two cases were expected to be symmetric about the center plane of the orifice. The CFDVAL case was computed with a full-plane grid, and results indeed maintained symmetry as expected. For the NASA Glenn case, use of a full-plane grid caused asymmetries to develop while establishing an initial steady-state flow field with constant blowing. The cause of these asymmetries is not precisely known, but it appears to be related to the strength of the blowing. Recall that the jet in the NASA Glenn case was strong enough to penetrate through the boundary layer to the freestream. If the jet strength was lowered significantly, then the full-plane constant blowing flow field remained symmetric. In any case, even with asymmetric initial conditions, these asymmetries went away and the solution became symmetric after the cyclic time-dependent boundary condition, to be described in the next section, was initiated.

\section{Results}

For the CFDVAL case, flow passed in and out of a circular orifice $D=6.35 \mathrm{~mm}$ in diameter. The orifice was located on the floor of a wind tunnel splitter plate with a turbulent boundary layer at $\mathrm{M}=0.1\left(U_{\infty}=34.6 \mathrm{~m} / \mathrm{s}\right)$ and approximate boundary layer thickness of $21 \mathrm{~mm}(\delta / D=3.3)$. The Reynolds number was 2230 per mm, or 14,160 per orifice diameter. The jet was driven electro-mechanically by a bottom-mounted square-shaped rigid piston mounted on an elastic membrane inside the cavity chamber beneath the splitter plate. The cavity was approximately $1.7 \mathrm{~mm}$ deep on average while the piston was operating, and the piston moved approximately $\pm 0.77 \mathrm{~mm}$. The thin volume inside the cavity varied during the operation of the piston, from roughly $1.0 \times 10^{4}-2.6 \times 10^{4} \mathrm{~mm}^{3}$. The frequency was $150 \mathrm{~Hz}$, and the maximum velocity $\left(w_{\max }\right)$ out of the orifice was approximately $1.3 U_{\infty}$. The momentum flux ratio, defined as $\left(w_{\max } / U_{\infty}\right)^{2}$, was approximately 1.69 . In this case, the effects due to the jet were mostly contained within the boundary layer. Experimental data were measured using laser-doppler velocimetry (LDV) and particle image velocimetry (PIV). ${ }^{16}$

For the NASA Glenn case, flow passed in and out of a circular orifice $D=19.05 \mathrm{~mm}$ in diameter. The Mach number of the freestream was $\mathrm{M}=0.0175\left(U_{\infty}=6.1 \mathrm{~m} / \mathrm{s}\right)$, and the turbulent boundary layer thickness was approximately $7.6 \mathrm{~mm}(\delta / D=0.4)$. The Reynolds number was 395.3 per $\mathrm{mm}$, or 7530 per orifice diameter. The jet was driven by a loud speaker at the bottom of a cavity mounted beneath the tunnel floor. The cavity was $152 \mathrm{~mm}$ deep (with a volume of approximately $2.8 \times 10^{7} \mathrm{~mm}^{3}$ ). The frequency was $24 \mathrm{~Hz}$, the maximum velocity out of the orifice was approximately $1.0 U_{\infty}$, and the momentum flux ratio was approximately 1.0. In this case the effects due to the jet extended well beyond the edge of the boundary layer. Experimental data were measured using hot-wire anemometry. ${ }^{17}$

For all of the results to follow, the $x$-direction is downstream and $z$-direction is up. The $y=0$ plane represents the center plane aligned with the center of the orifice. Two different gridding approaches were taken for modeling the two cases. Because the CFDVAL case employed such a shallow cavity, the computational grid included the (as-built) cavity. A time-dependent velocity boundary condition was applied at the (fixed) bottom of the cavity to simulate the effect of a moving wall. Additional details can be found in Rumsey. ${ }^{22}$ For the NASA Glenn case, the cavity was so large relative to the orifice that it was impractical to model it in the computations. Therefore, in this case the grid included the orifice only, which extended $25.4 \mathrm{~mm}$ below the tunnel floor. For both cases, the initial conditions were steady-state flow with constant blowing. Subsequently, a time-dependent velocity boundary condition was applied at the bottom of the orifice or cavity. This boundary condition was calibrated in order to attempt to match the velocity as a function of phase at the orifice exit, as measured experimentally. The time-dependent velocities prescribed were:

$$
u=0 \quad v=0 \quad w=\left[(\rho w)_{\max } / \rho\right] \cos (2 \pi F t)
$$

where $(\rho w)_{\max }=0.0008 \rho_{\infty} a_{\infty}$ for the CFDVAL case, and $0.0175 \rho_{\infty} a_{\infty}$ for the NASA Glenn case. $F$ was the frequency of oscillation. Due to the very low Mach number of the NASA Glenn case, preconditioning was employed in that particular computation. At the upstream grid boundary, a turbulent profile was specified that matched the corresponding experimental incoming boundary layer profile.

Figure 2 shows time history of the velocities near the center of the orifice for the CFDVAL case. Results are shown for two different full-plane grids (the fine grid had 4.1 million total points and the medium grid was created using every other point from the fine grid), and for three different turbulence models (SA=Spalart-Allmaras, SST=Menter's shear stress transport $k-\omega$, and EASM=explicit algebraic stress model). For the most part differences due to grid and turbulence model were relatively small. The CFD mimicked the general overall trends measured in the experiment, but there were some noticeable specific differences. However, these results were the best it was possible to attain within the constraints imposed by the modeling and boundary conditions used. See Rumsey ${ }^{22}$ for more discussion on these 
differences.

Figure 3 shows long-time average $u$-velocity contours for the CFDVAL case on the center plane using CFD with the SA model. Although not shown, results from the other turbulence models looked very similar. The mean perturbation to the velocity field was mostly contained within the boundary layer. There were not enough experimental data available to cover the same plotting range as Fig. 3, but Fig. 4 shows a comparison between CFD and experiment for $u$-velocity contours in a zoomed-in region very near the orifice exit, where $x_{0}$ is the orifice center. There was only a crude qualitative correspondence between the two. The CFD result predicted a larger mean perturbation to the flow field (including a very small reverse flow region near the orifice lip at $\left(x-x_{0}\right) / D=0.5$ that is difficult to see in the figure), whereas the experimental PIV data showed the retarded velocity region to be smaller and offset downstream from the lip. Other results for the CFDVAL case can be found in Rumsey. ${ }^{22}$ Generally CFD was successful in qualitatively predicting many flow features - both long-time-averaged and phase-averaged - compared with LDV measurements.

For the NASA Glenn case, only one turbulence model, SA, was employed. As discussed in section II, there were some problems maintaining symmetry on a full-plane grid when establishing the constant blowing initial condition, but subsequent unsteady computations re-established symmetry. The effect of computing with a full-plane grid vs. a half-plane grid was investigated for this case. Results showed very little difference, so all NASA Glenn results for the remainder of the paper were obtained using the half-plane grid only. A sample comparison showing long-time average $u$-velocity contours in a plane parallel to the floor is shown in Fig. 5.

Figure 6 shows the fine half-plane grid, made up of 1.8 million points. This grid had the same (rescaled) distribution of grid points as the CFDVAL case outside of the orifice. A medium grid was also employed that was created using every other point from the fine grid. The time history of velocity at the orifice center is shown in Fig. 7. Two different grid sizes made very little difference in the results. The total velocity agreed fairly well with the experiment, which did not measure the individual velocity components like in the CFDVAL case. The largest discrepancy between CFD and experiment was in the magnitude during the suction part of the cycle. The computations accurately captured the double hump in total velocity during the discharge part of the cycle seen in the experiment.

These results were obtained using a time step corresponding to 720 steps per cycle, and 10 subiterations per time step. The effects of time step and number of subiterations were also investigated for the NASA Glenn case. Total velocity at the orifice center is plotted in Fig. 8 for the three conditions of 720 steps per cycle and 10 subiterations, 720 steps per cycle and 20 subiterations, and 360 steps per cycle and 10 subiterations. These results were obtained on the medium grid. There were imperceptible differences between the three results. Contour plots in the flow field also indicated a high degree of temporal convergence. For example, Fig. 9 shows phase-averaged $u$-velocity contours in the plane $z=0.192 \mathrm{~mm}$ at phase $=0^{\circ}$. The left half of the figure shows results using 360 steps per period and the right half shows results using 720 steps per period.

Figure 10 shows long-time average contours of $u / U_{\infty}$ in the center plane for the NASA Glenn case. The agreement between CFD and experiment was qualitatively very good. The mean perturbation to the velocity field in this case extended well beyond the boundary layer edge. Phase averaged contour plots (not shown here, but given in Milanovic et al. ${ }^{17}$ ) also showed good qualitative agreement. In particular, CFD captured the size and position of the disturbance very well as it convected down the plate after being emitted from the orifice, although the tilt in the structures predicted by the CFD was less pronounced than in the experiment.

Quantitative comparisons of $u$-velocity profiles as a function of phase at a fixed position $\left(x-x_{0}\right) / D=2.235$, $y=0$ are shown in Fig. 11. From Milanovic et al., ${ }^{17}$ the convection speed of the synthetic jet structure created during the peak expulsion part of the cycle (phase $\approx 260^{\circ}$ ) was approximately $0.75 U_{\infty}$. Thus, the structure convected to $\left(x-x_{0}\right) / D=2.235$ at approximately phase $=340^{\circ}$. As the figure shows, at the two phases $320^{\circ}$ and $0^{\circ}$ surrounding this "event," the velocity profiles were significantly affected, and the CFD predicted perturbed velocities in the boundary layer that were too low compared with experiment. However, the profile shapes were qualitatively similar, and the profiles at the other three phases were quantitatively predicted very well. The long-time average also yielded excellent agreement. Comparisons of phase-averaged profiles for the CFDVAL case were reported in Rumsey. ${ }^{22}$ Overall, the agreement for the CFDVAL case was generally better than for the NASA Glenn case during the "event" portion of the cycle. Figure 11 also compares results on both the fine and medium grids, giving an indication of the approximate effect of discretization error for these grid sizes. The effect was relatively small.

Figures 12 and 13 are contour plots showing the vertical velocity at the orifice for both the CFDVAL and NASA Glenn cases, within $20^{\circ}$ of peak discharge and peak suction. Note that for the two cases the phases (which were designated by the individual experiments) were not the same: for the CFDVAL case peak discharge occurred near phase $=120^{\circ}$ and peak suction near phase $=300^{\circ}$, whereas for the NASA Glenn case peak discharge occurred near phase $=260^{\circ}$ and peak suction near phase $=80^{\circ}$. However, this phase designation is arbitrary, and in the figure 
the closest phase available from the stored CFD data is displayed. Both cases showed similar behavior. Near peak discharge, the velocities out of the orifice were very nonuniform, and the strongest velocities occurred near the side edges of the orifice, downstream of center. Near peak suction, the velocities were more uniform over much of the orifice area. These figures indicate the importance of modeling the inside of the orifice in CFD computations. Although a simple time-dependent top-hat profile might adequately model the relatively uniform inflow during the suction phase, it would be difficult to prescribe a time-dependent profile at the exit plane that mimicked the non-uniform velocities present during the expulsion part of the cycle. The effect of ignoring the inside of the orifice, and instead applying the time dependent boundary condition (Eq. 11) directly at the orifice exit, was investigated. Quantitative comparisons are given in Fig. 14, showing both phase-average results as well as the long-time average. Not modeling the inside of the orifice had an overall negative impact on the comparison with experiment, as expected.

\section{Conclusions}

Time-dependent Reynolds-averaged Navier-Stokes computations were described for two different synthetic jet flows issuing into a turbulent boundary layer crossflow through a circular orifice. In one case the jet effect was mostly contained within the boundary layer, while in the other case the jet effect extended beyond the boundary layer edge. The former case was originally one of the subjects of the CFDVAL2004 workshop on synthetic jets.

The use of URANS was shown to be appropriate and viable for synthetic jet flows. In both cases described here, the computations did a reasonably good job predicting the qualitative behavior of the synthetic jet flow field. However, quantitatively there were many specific differences. Several numerical parameters were investigated, including effect of grid size, time step, number of subiterations, and turbulence model. Differences between results on the fine and medium grids were relatively small. Using at least 360 time steps per period and at least 10 subiterations per time step was more than sufficient to produce results that did not change perceptibly with further temporal refinement. Different turbulence models for the CFDVAL case had some influence on the solution, but these differences were generally not too large.

For synthetic jets like those considered here, either a full-plane grid or a half-plane grid (with symmetry) could be used. The current computations showed the importance of including the region inside the orifice when modeling the problem. Use of time-dependent top-hat boundary conditions at the orifice exit plane was an oversimplification that failed to capture the complex nature of the flow field near the orifice, particularly during the expulsion phase of the cycle.

\section{References}

${ }^{1}$ Seifert, A., Bachar, T., Koss, D., Shepshelovich, M., and Wygnanski, I., "Oscillatory Blowing: A Tool to Delay Boundary-Layer Separation," AIAA J., Vol. 31, No. 11, 1993, pp. 2052-2060.

${ }^{2}$ Gordon, M. and Soria, J., "PIV Measurements of a Zero-Net-Mass-Flux Jet in Cross Flow," Exp. Fluids, Vol. 33, 2002, pp. 863-872.

${ }^{3}$ Smith, D. R., "Interaction of a Synthetic Jet with a Crossflow Boundary Layer," AIAA J., Vol. 40, No. 11, 2002, pp. $2277-2288$.

${ }^{4}$ Milanovic, I. M. and Zaman, K. B. M. Q., "Synthetic Jets in Crossflow," AIAA J., Vol. 43, No. 5, 2005, pp. 929-940.

${ }^{5}$ Sauerwein, S. C. and Vakili, A. D., “An Experimental Study of Zero-Mass Jets in Crossflow,” AIAA Paper 99-0668, January 1999.

${ }^{6}$ Lee, C. Y. and Goldstein, D. B., "DNS of Microjets for Turbulent Boundary Layer Control," AIAA Paper 2001-1013, January 2001.

${ }^{7}$ Mittal, R., Rampunggoon, P., and Udaykumar, H. S., "Interaction of a Synthetic Jet with a Flat Plate Boundary Layer," AIAA Paper 20012773, June 2001.

${ }^{8}$ Mittal, R. and Rampunggoon, P., "On the Virtual Aeroshaping Effect of Synthetic Jets," Phys. Fluids, Vol. 14, No. 4, 2002, pp. $1533-1536$.

${ }^{9}$ Ravi, B. R., Mittal, R., and Najjar, F. M., "Study of Three-Dimensional Synthetic Jet Flowfields Using Direct Numerical Simulation,” AIAA Paper 2004-0091, January, 2004.

${ }^{10}$ Cui, J., Agarwal, R. K., and Cary, A. W., "Numerical Simulation of the Interaction of a Synthetic Jet with a Turbulent Boundary Layer," AIAA Paper 2003-3458, June 2003.

${ }^{11}$ Cui, J. and Agarwal, R. K., "3-D CFD Validation of an Axisymmetric Jet in Cross-Flow (NASA Langley Workshop Validation: Case 2)," AIAA Paper 2005-1112, January 2005.

${ }^{12}$ Rizzetta, D. P., Visbal, M. R., and Stanek, M. J., "Numerical Investigation of Synthetic-Jet Flowfields," AIAA J., Vol. 37, No. 8, 1999, pp. 919-927.

${ }^{13}$ Spalart, P. R., and Allmaras, S. R., "A One-Equation Turbulence Model for Aerodynamic Flows," La Recherche Aerospatiale, No. 1, 1994, pp. 5-21.

${ }^{14}$ Sellers, W. L. and Rumsey, C. L., "Langley Research Center Workshop: CFD Validation of Synthetic Jets and Turbulent Separation Control," (online), URL: http://cfdval2004.larc.nasa.gov (cited 4 May 2005).

${ }^{15}$ Rumsey, C. L., Gatski, T. B., Sellers, W. L. III, Vatsa, V. N., and Viken, S. A., "Summary of the 2004 CFD Validation Workshop on Synthetic Jets and Turbulent Separation Control,” AIAA Paper 2004-2217, June-July 2004.

${ }^{16}$ Schaeffler, N. W. and Jenkins, L. N., "The Isolated Synthetic Jet in Crossflow: A Benchmark for Flow Control Simulation," AIAA Paper 2004-2219, June-July 2004. 
${ }^{17}$ Milanovic, I. M., Zaman, K. B. M. Q., Rumsey, C. L., “An Isolated circular Synthetic Jet in Crossflow at Low Momentum-Flux Ratio," AIAA Paper 2005-1110, January, 2005.

${ }^{18}$ Menter, F. R., “Two-Equation Eddy-Viscosity Turbulence Models for Engineering Applications," AIAA Journal, Vol. 32, No. 8, 1994, pp. 1598-1605.

${ }^{19}$ Spalart, P. R., Jou, W. H., Strelets, M., and Allmaras, S. R., "Comments on the Feasibility of LES for Wings, and on a Hybrid RANS/LES Approach," First AFOSR International Conference on DNS/LES, in Advances in DNS/LES, edited by C. Liu and Z. Liu, Greyden Press, Columbus, $\mathrm{OH}, 1997$.

${ }^{20}$ Krist, S. L., Biedron, R. T., and Rumsey, C. L., “CFL3D User’s Manual (Version 5.0),” NASA TM-1998-208444, June 1998.

${ }^{21}$ Rumsey, C. L., "Reynolds-Averaged Navier-Stokes Computations of a Synthetic Jet in a Turbulent Boundary Layer," Proceedings of ICCFD3, July 2004, Toronto, CANADA, to appear.

${ }^{22}$ Rumsey, C. L., "Computation of a Synthetic Jet in a Turbulent Cross-Flow Boundary Layer," NASA/TM-2004-213273, October 2004.

${ }^{23}$ Weiss, J. M., and Smith, W. A., "Preconditioning Applied to Variable and Constant Density Flows," AIAA Journal, Vol. 33, No. 11, 1995, pp. 2050-2057.

${ }^{24}$ Roe, P. L., “Approximate Riemann Solvers, Parameter Vectors, and Difference Schemes,” J. Comp. Phys., Vol. 43, 1981, pp. 357-372.

${ }^{25}$ Rumsey, C. L. and Gatski, T. B., "Summary of EASM Turbulence Models in CFL3D with Validation Test Cases," NASA/TM-2003-212431, June 2003.

${ }^{26}$ Reynolds, W. C. and Hussain, A. K. M. F., "The Mechanics of an Organized Wave in Turbulent Shear Flow. Part 3. Theoretical Models and Comparisons with Experiment," J. Fluid Mech., Vol. 54, Part 2, 1972, pp. 263-288.

${ }^{27}$ Gatski, T. B. and Liu, J. T., "On the Interactions between Large-Scale Structure and Fine-Grained Turbulence in a Free Shear Flow. III. A Numerical Solution,” Phil. Trans. Roy. Soc. London, Vol. 293, 1980, pp. 473-509.

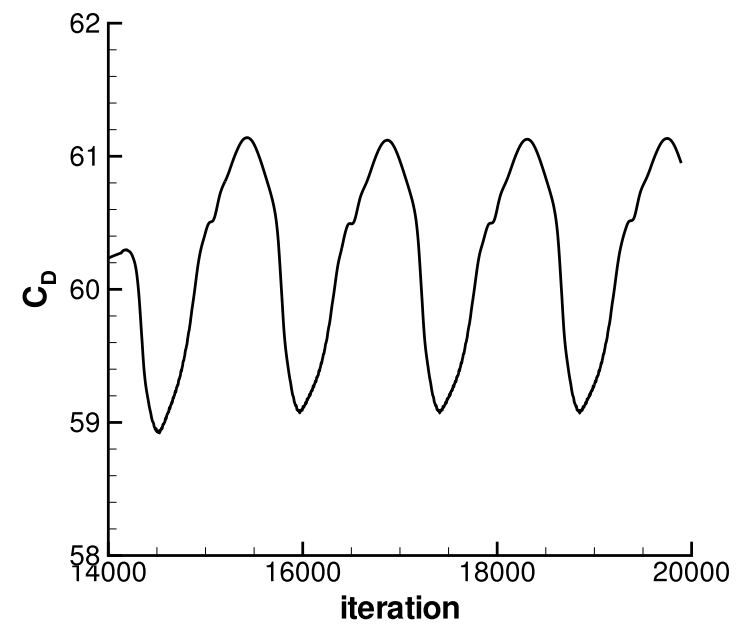

Figure 1. Scaled drag coefficient as a function of iteration, showing periodic repeatability of URANS computation for CFDVAL case. 

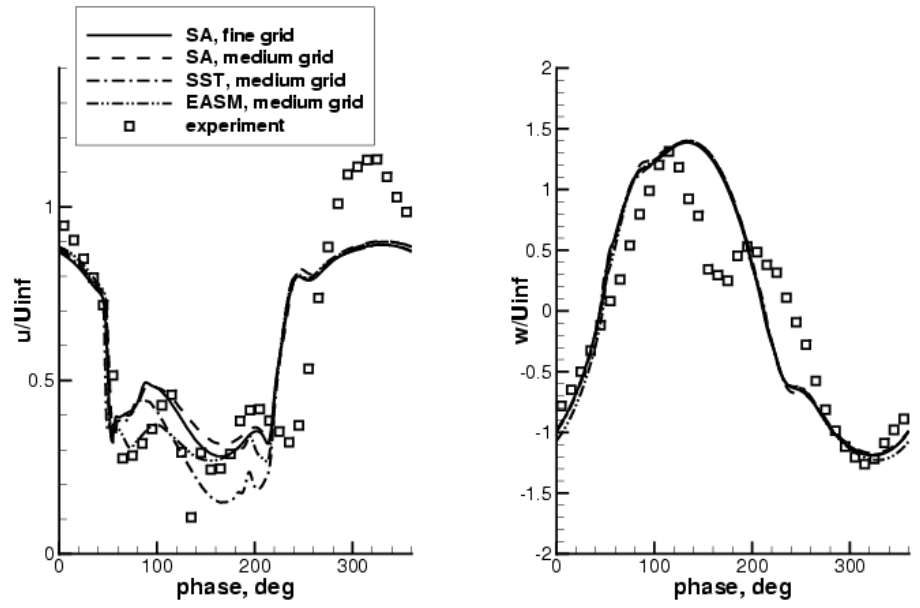

Figure 2. Time history of velocity near center of orifice for CFDVAL case ( $z=0.4 \mathbf{~ m m}$ above the floor).

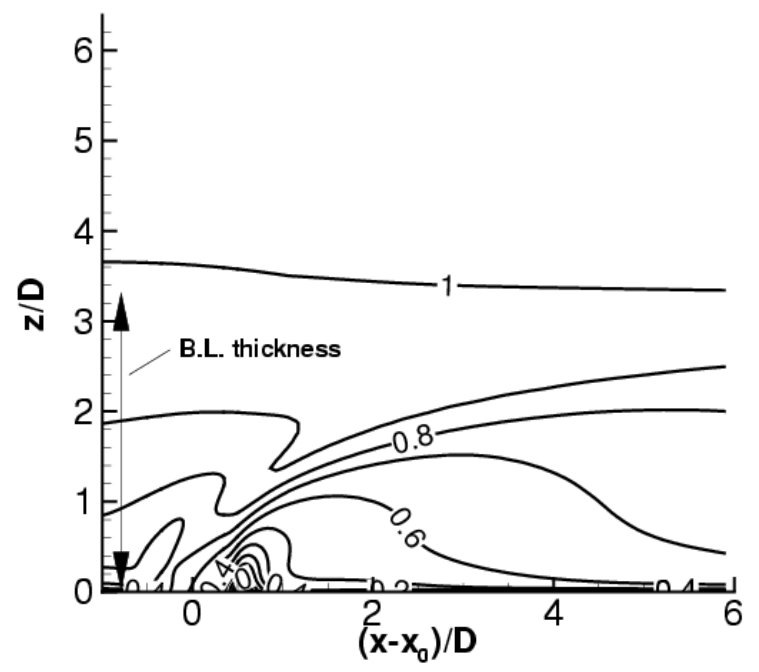

Figure 3. Computed long-time-average $u / U_{\infty}$ contours in center plane of CFDVAL case (orifice is centered at $\left.\left(x-x_{0}\right) / D=0\right)$, SA, fine grid. 

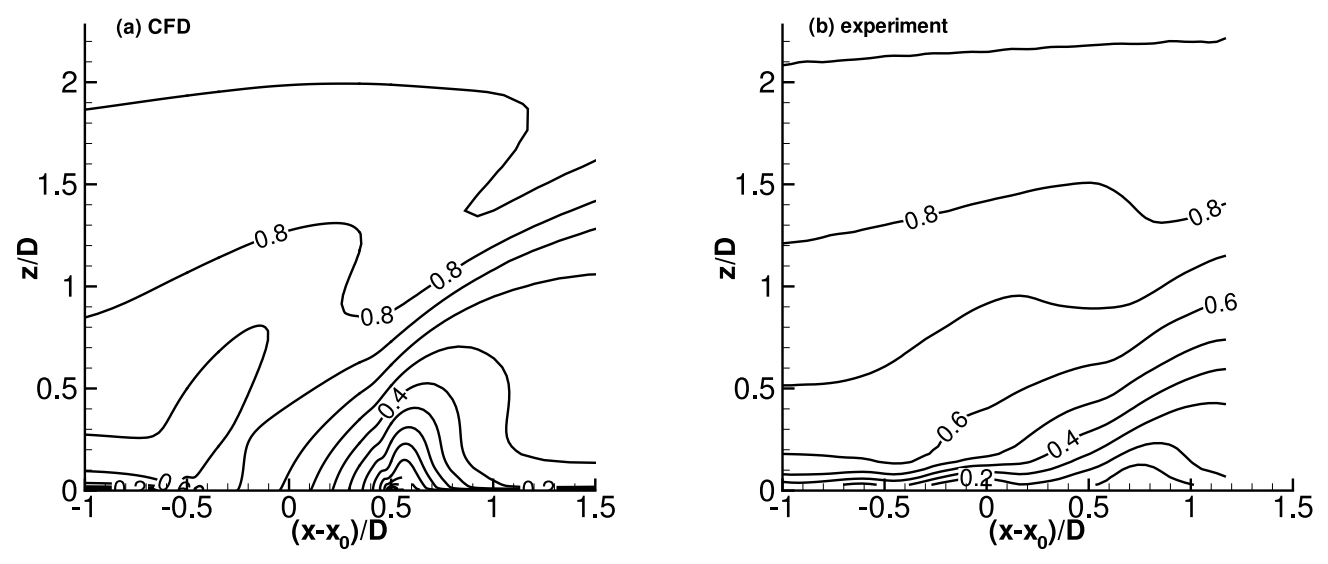

Figure 4. Close-up of long-time-average $u / U_{\infty}$ contours in center plane of CFDVAL case near orifice; (a) CFD (SA, fine grid), (b) experiment (PIV).

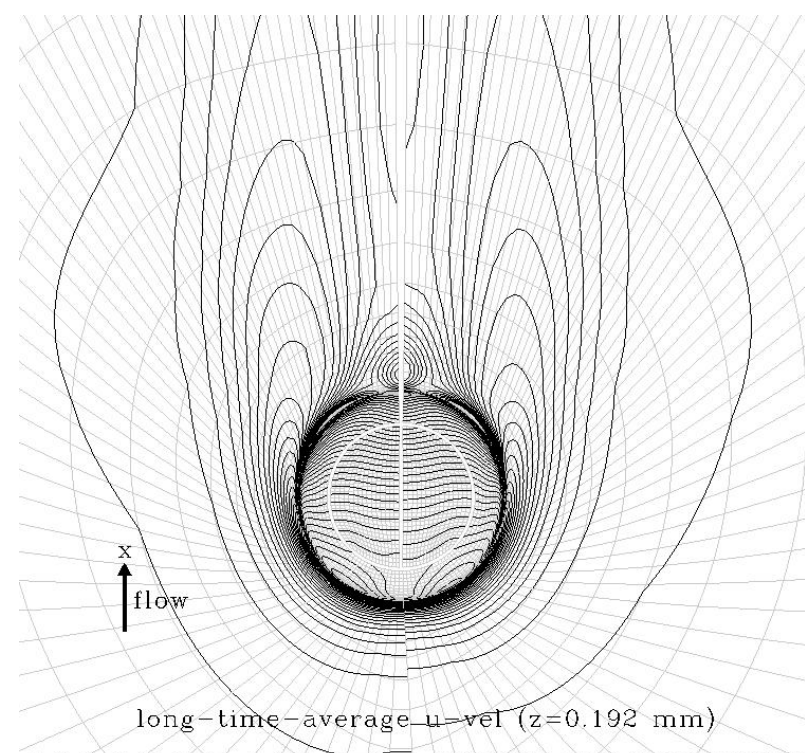

Figure 5. Contours of long-time average $u$-velocity in the $z=0.192 \mathbf{m m}$ plane for NASA Glenn case using SA, medium grids, showing difference between full-plane computations (left half) and half-plane with symmetry (right half). 


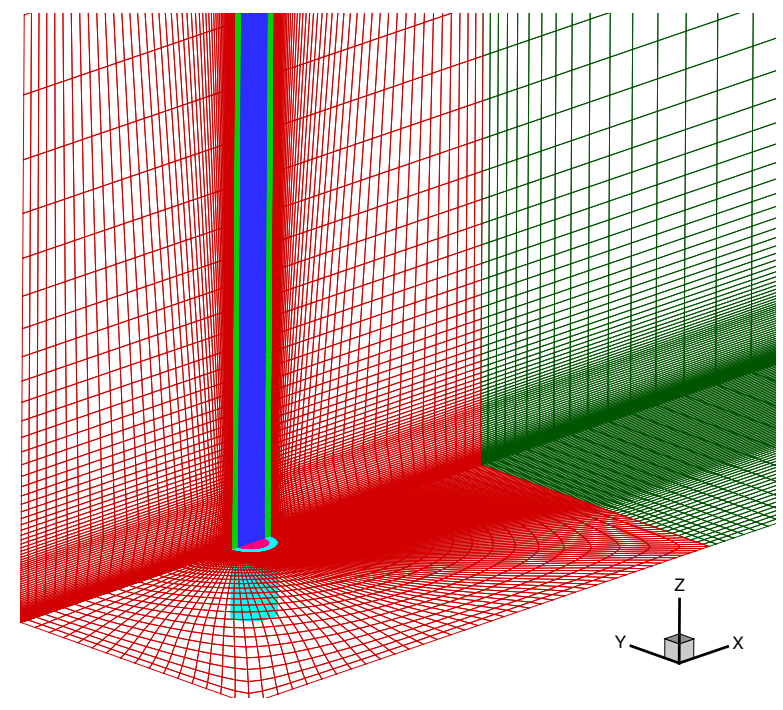

Figure 6. Picture of the half-plane 6-zone grid used for NASA Glenn case (1.8 million grid points total).

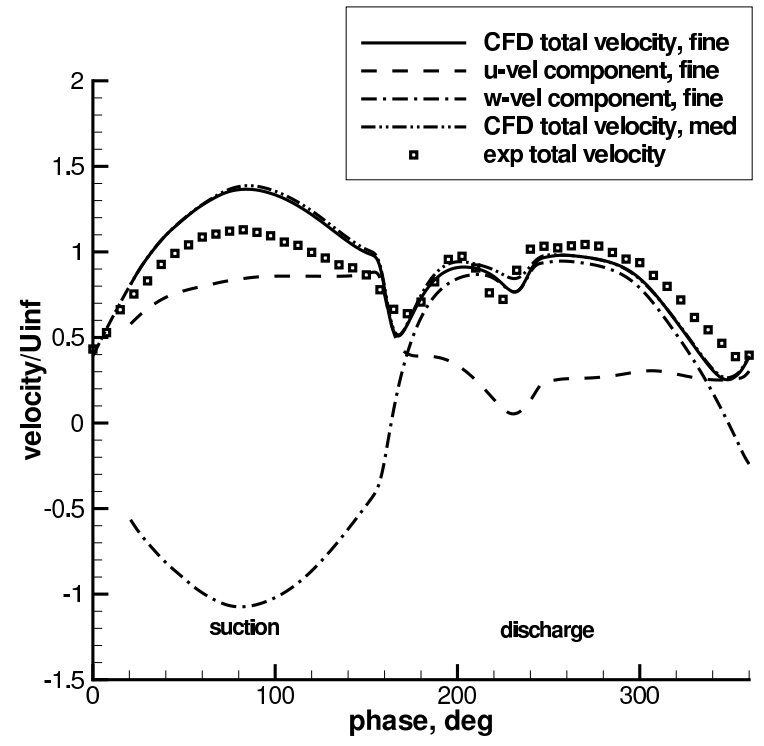

Figure 7. Time history of velocity at center of orifice for NASA Glenn case, SA. 


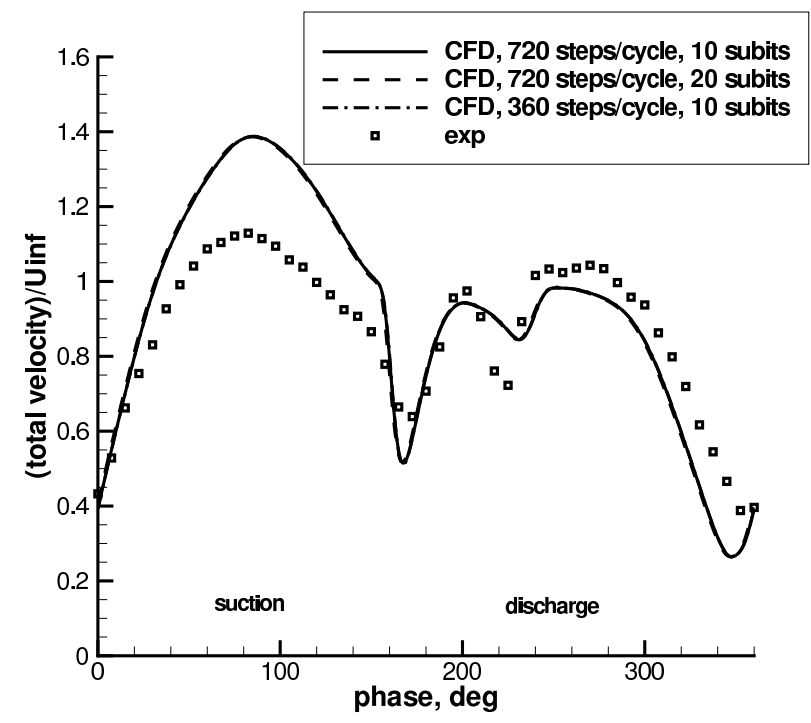

Figure 8. Effect of time step and number of subiterations on time history of total velocity at center of orifice for NASA Glenn case, SA, medium grid.

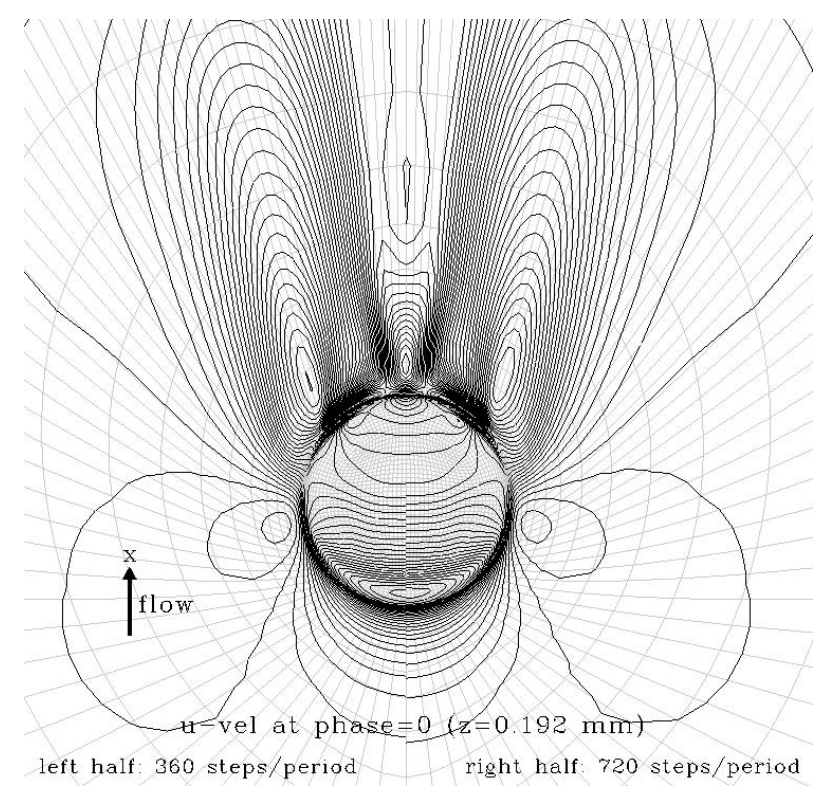

Figure 9. Contours of $u$-velocity at phase $=0^{\circ}$ in the $z=0.192 \mathrm{~mm}$ plane for NASA Glenn case using SA, medium grid, showing difference between 360 steps per period (left half) and 720 steps per period (right half). 

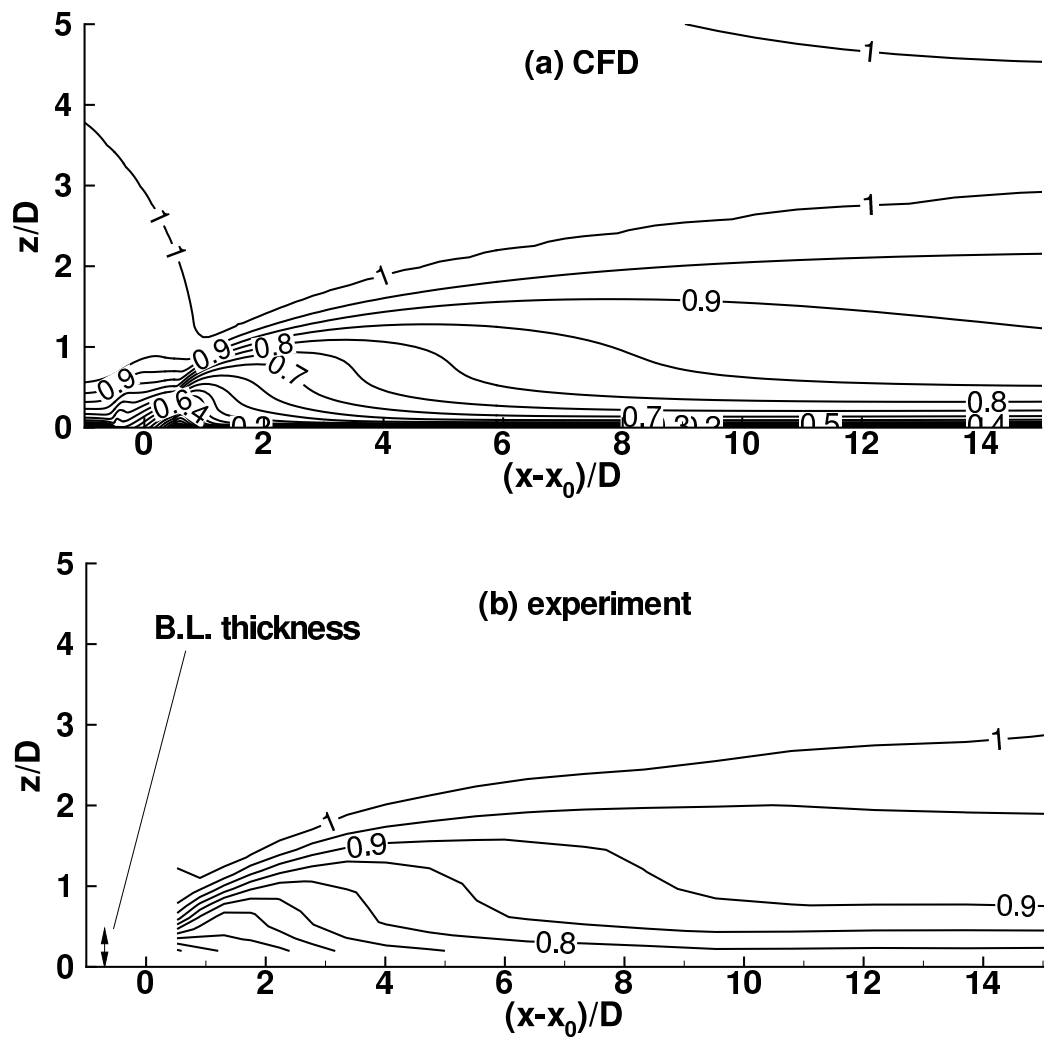

Figure 10. Comparison of long-time average $u / U_{\infty}$ contours in center plane of NASA Glenn case (orifice is centered at $\left(x-x_{0}\right) / D=0$ ), SA, fine grid. 

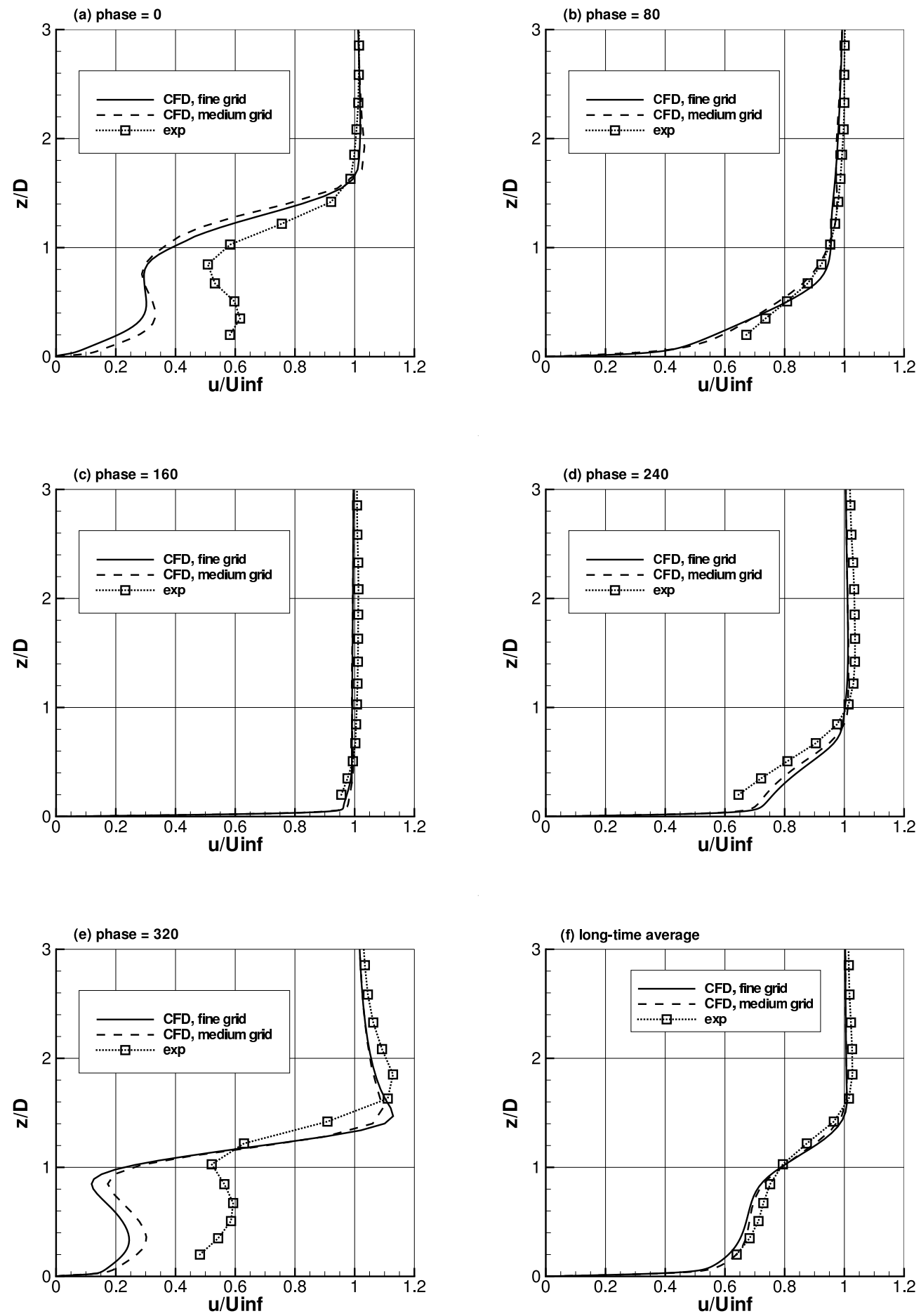

Figure 11. Profiles of $u$-velocity at $\left(x-x_{0}\right) / D=2.235, y=0$ as a function of phase and long-time average for NASA Glenn case, SA. 

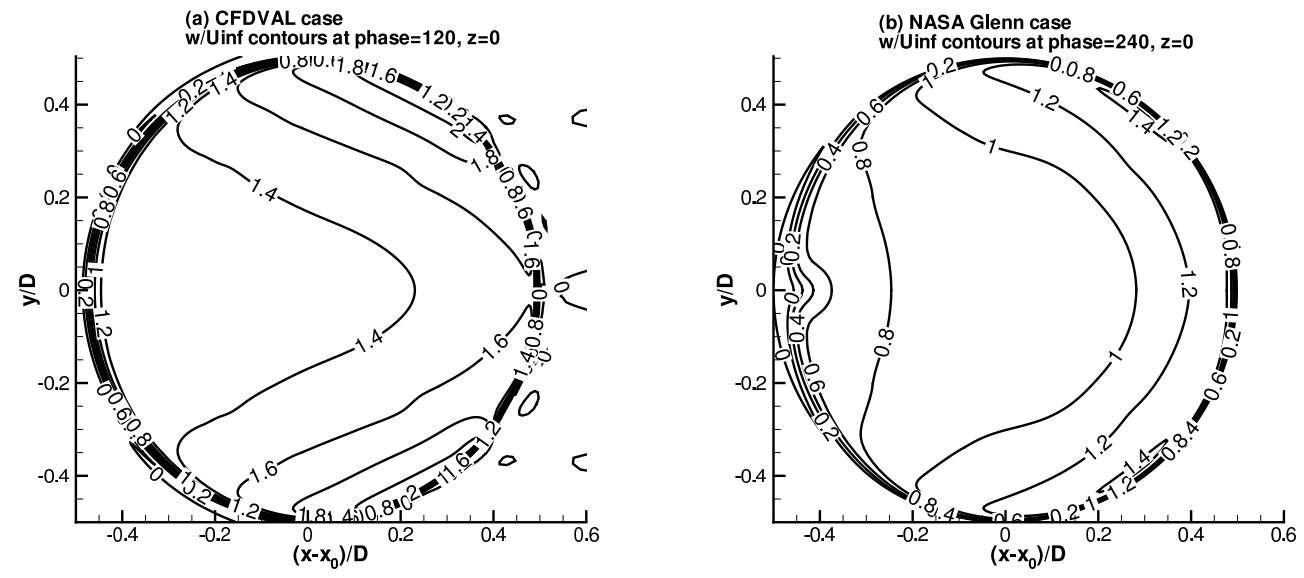

Figure 12. Contours of $w$-velocity at orifice exit plane near peak discharge part of cycle using SA, fine grid; CFDVAL case on left, NASA Glenn case on right.
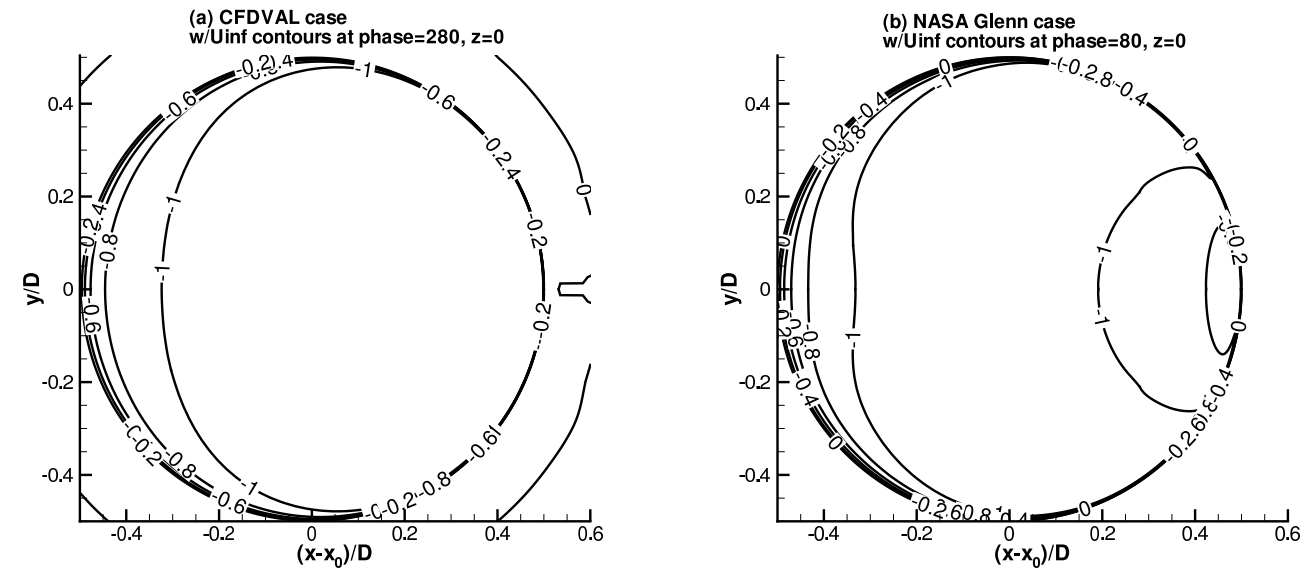

Figure 13. Contours of $w$-velocity at orifice exit plane near peak suction part of cycle using SA, fine grid; CFDVAL case on left, NASA Glenn case on right. 

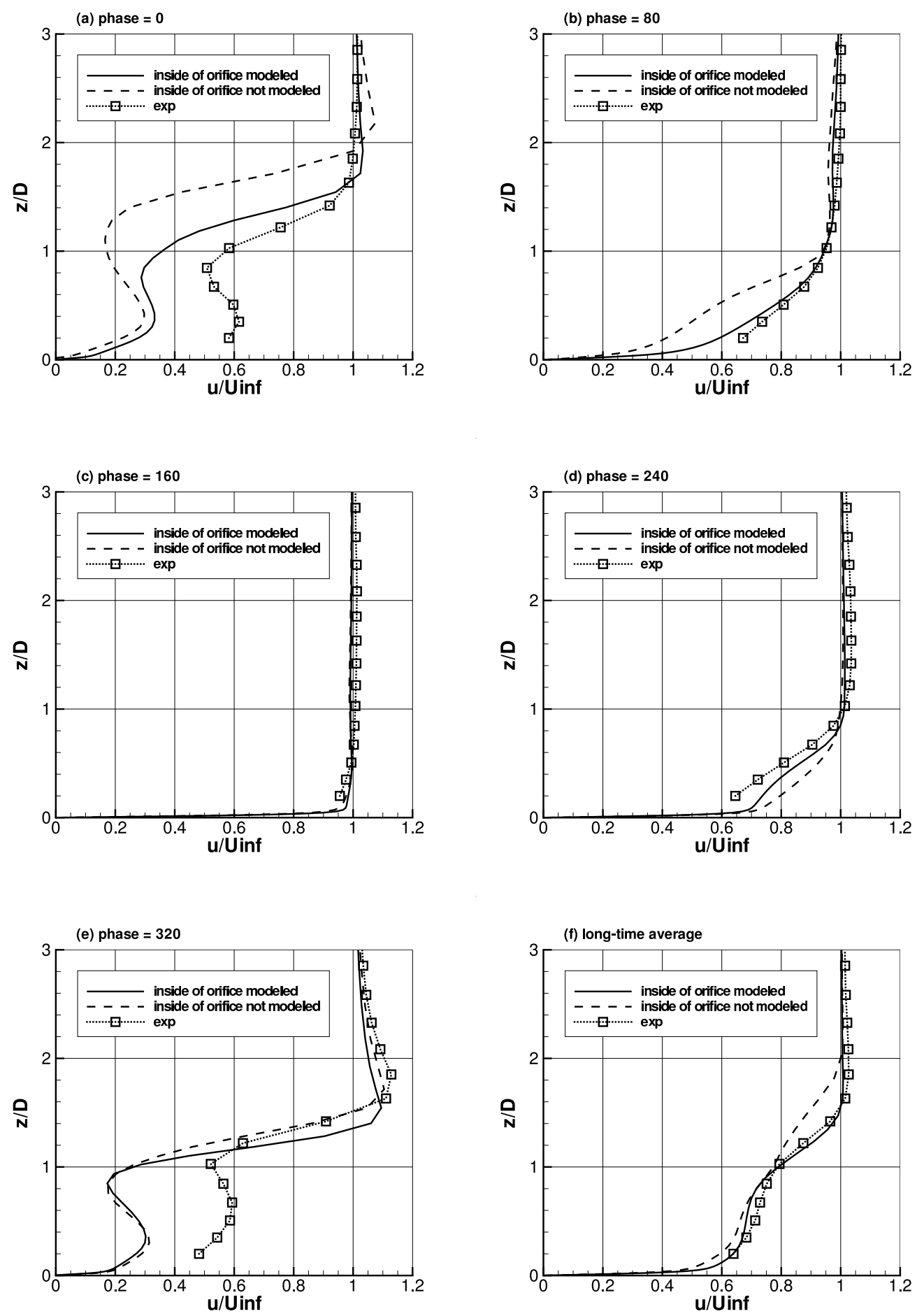

Figure 14. Effect of orifice treatment on phase-average and long-time-average $u$-velocity profiles at $\left(x-x_{0}\right) / D=2.235, y=0$ for NASA Glenn case, SA, medium grid. 